\title{
Urinary fluorescence analysis in diagnosis of bladder cancer
}

\author{
B. KOLLARIK ${ }^{1, *}$, M. ZVARIK ${ }^{2}$, P. BUJDAK ${ }^{3}$, P. WEIBL ${ }^{4}$, L. RYBAR$^{1}$, L. SIKUROVA ${ }^{2}$, L. HUNAKOVA ${ }^{5}$ \\ ${ }^{1}$ Department of Urology, University Hospital of Bratislava, Slovakia; ${ }^{2}$ Department of Nuclear Physics and Biophysics, Faculty of Mathematics, \\ Physics and Computer Science, Comenius University, Bratislava, Slovakia; ${ }^{3}$ Slovak Medical University, Bratislava, Slovakia; ${ }^{4}$ UROclinic s.r.o. \\ Bratislava, Slovakia; ${ }^{5}$ Cancer Research Institute BMC, Slovak Academy of Sciences, Bratislava, Slovakia
}

${ }^{*}$ Correspondence: bkollarik@gmail.com

Received June 10, 2017/ Accepted July 12, 2017

\begin{abstract}
Early diagnosis of bladder cancer is crucial for improvement of cancer specific survival and recurrence rate. We analyzed the possible role of fluorescence urine analysis in bladder cancer diagnosis. The cohort consisted of 20 healthy controls, 40 patients with hematuria and 75 patients with hematuria and histologically proven bladder tumor. Synchronous fluorescence spectra with a $70 \mathrm{~nm}$ wavelength difference were recorded for (1:1-1:128) urine dilutions. Concentration matrices of synchronous spectra (CMSS) were used to classify samples into tested groups. CMSS analysis allowed us to distinguish patients with tumor from patients with hematuria with a sensitivity $55 \%$ and specificity $74.7 \%$. This is comparable to the sensitivity and specificity of other non-invasive tests like BTA stat and nmP-22 (Bladder check ${ }^{\circledR}$ ). Lower fluorescence intensity of Imax $280 \mathrm{~nm}$ and ratio of $280 \mathrm{~nm}$ to $450 \mathrm{~nm}$ was found to be associated with the presence of tumor. We have found an association of decreased fluorescence with the stage of the disease. Our data suggest that CMSS urine analysis has a potential role in the non-invasive diagnostic tests for bladder cancer, but it cannot replace the current diagnostic algorithm yet.
\end{abstract}

Key words: bladder cancer, hematuria, fluorescence spectra, urine, metabolite

Bladder cancer belongs to one of the most common malignancies with approximately 430000 new cases per year and high mortality of 165000 cases [1]. One fourth of the patients is still diagnosed with muscle invasive disease. Bladder cancer has a high risk of recurrence, reported $50-70 \%$ and up to $15 \%$ of patients progress during the follow up $[1,2]$. The diagnosis and follow up of bladder cancer is based on cytology and cystoscopy. There is a need for new non-invasive markers for early diagnosis and follow up of bladder cancer; cystoscopy is a costly and invasive procedure with excellent sensitivity and specificity. Cytology as the only widely used non-invasive test has a low sensitivity especially for the low-grade tumors. The currently known non-invasive tests for bladder cancer have acceptable sensitivity, but a high grade of false positivity, which makes it difficult to use them for diagnosis and follow up of urothelial bladder cancer [3].

Urine is an aqueous solution of a variety of organic and anorganic compounds, including a number of natural fluorophores, most of which are metabolites of tryptophan $[4,5]$. Spectroscopic urine analysis is based on the measurement of fluorescence of the metabolites after excitation with a light source of a certain wavelength. Fluorescence is an optical phenomenon caused by a shift of an electron from excitation state to a stable state. The published studies on spectroscopic urine analysis show a high sensitivity and specificity for urothelial bladder cancer [6]. Also Martinicky et al. suggest a potential role of spectroscopic urine analysis in diagnosis and screening of ovarial carcinoma [7]. In this study, we investigated the possible diagnostic potential of human urine autofluorescence analysis, its ability to distinguish between hematuria and patients with tumors and hematuria, and the correlation of spectral changes with known prognostic factors - grade and stage, as well as comparison with cytology and other non-invasive tests.

\section{Patients and methods}

Patient characteristics. This study was approved by the University Hospital of Bratislava Ethics Review Board for human studies. All the patients signed informed consent prior to the study enrollment, according to the World Medical Association Declaration of Helsinki. Patients included in the study underwent recommended non-invasive tests (urine for cytology, urine cultivation). Urine for cytology 
was taken according to the recommended protocol and immediately fixed with formaldehyde 1:1. Fasting morning urine samples for spectroscopic analysis and cultivation were obtained. All of the hematuria patients underwent cystoscopy, in all patients with a suspect finding in cystoscopy or positive cytology, histology of the resected tissue was performed which is in concordance with the EAU guidelines on bladder cancer. The patients were divided into subgroups according to hematuria and histological results, healthy control (consisted of 20 patients with normal laboratory and cytologic findings), patients with hematuria tumor negative (40 patients) and patients with tumor and positive hematuria (75 patients). None of the patients in the study had a positive urine cultivation, histological examination of the removed tissue confirmed malignant urothelial bladder tumors in 75 patients. Demographic characteristics of patients are shown in Table 1.

Fluorescence analysis of urine. Urine samples were centrifuged at $3000 \mathrm{rpm}$ for $10 \mathrm{~min}$ at room temperature $\left(22 \pm 1^{\circ} \mathrm{C}\right)$ and undiluted supernatants were used for spectral analysis. The detailed fluorescence data of urine samples were obtained on a LS45 (PerkinElmer) luminescence spectrometer using the FL WinLab software. Measurements were taken at room temperature $\left(22 \pm 1^{\circ} \mathrm{C}\right)$ in an ultra-micro quartz cuvette $(5 \mathrm{~mm}$ excitation path length and $1 \mathrm{~mm}$ emission path length) in $100 \mu \mathrm{l}$ sample volume. Quality control samples including blank ones were run before the beginning of the experiment to ensure the stability of samples and the equipment. Both, the emission and excitation slit widths, were set to $10 \mathrm{~nm}$. Synchronous fluorescence spectra (SFS) were collected by simultaneously scanning the excitation and emission monochromator in the excitation wavelength range $250-550 \mathrm{~nm}$ with constant wavelength differences $\Delta \lambda=70 \mathrm{~nm}$ between them and in a broad range of urine dilutions (1:1-1:128). Concentration matrices of synchronous spectra (CMSS) represent the relations between the fluorescence intensity, excitation wavelength and urine dilution (fraction volume). They were created by the set of synchronous fluorescence spectra of urine samples diluted by geometrical progression. The $\mathrm{x}$-axis represents the excitation wavelength of synchronous fluorescence scanning and the $y$-axis the urine dilution (geometric progression). Contour lines connect regions of the same fluorescence intensity and each contour line represents 5 percent of the full fluorescence scale.

Statistical analysis. The collected data were statistically and graphically processed by the R software. For the correlation of the chosen spectral characteristics and the prognostic factors (histology, stage, grade) Mann Whitney test was performed. We made a box and whisker plot from these spectral characteristics, where the bottom and top of the box

Table 1. The demographic characteristics of patients.

\begin{tabular}{|c|c|c|c|c|}
\hline Patient demographics & HEALTHY & HEMATURIA & HEMATURIA/TU & ENTIRE COHORT \\
\hline Patients num. (\%) & $20(100 \%)$ & $40(100 \%)$ & $75(100 \%)$ & $136(100 \%)$ \\
\hline Males & $13(65 \%)$ & $24(60 \%)$ & $52(69.3 \%)$ & $90(66.2 \%)$ \\
\hline Females & $7(35 \%)$ & $16(40 \%)$ & $23(30.7 \%)$ & $46(33.8 \%)$ \\
\hline M:F ratio & $1.41: 1$ & $1.86: 1$ & $2.36: 1$ & $1.93: 1$ \\
\hline \multicolumn{5}{|l|}{ Age, years } \\
\hline Mean & 60.15 & 66.05 & 65.03 & 64.6 \\
\hline Median & 65 & 65 & 65.5 & 65 \\
\hline Range & $(31-75)$ & $(38-83)$ & $(22-89)$ & $(22-89)$ \\
\hline Smokers\% & $5(22 \%)$ & $12(30 \%)$ & $49(65.3 \%)$ & $66(48.9 \%)$ \\
\hline \multicolumn{5}{|l|}{ Hematuria } \\
\hline Macroscopic & $0(0 \%)$ & $23(57.5 \%)$ & $56(74.7 \%)$ & $79(58.5 \%)$ \\
\hline Microscopic & $0(0 \%)$ & $17(42.5 \%)$ & $19(25.3 \%)$ & $56(41.5 \%)$ \\
\hline \multicolumn{5}{|l|}{ Histolology } \\
\hline Urothelial tumors & $0(0 \%)$ & $0(0 \%)$ & $71(94.7 \%)$ & $71(52.6 \%)$ \\
\hline Urothelial tumors subtypes & $0(0 \%)$ & $0(0 \%)$ & $4(5.3 \%)$ & $4(3 \%)$ \\
\hline Non-urothelial tumors & $0(0 \%)$ & $0(0 \%)$ & 1 (excluded) & 1 (excluded) \\
\hline \multicolumn{5}{|l|}{ Grade, 2004 WHO } \\
\hline low-grade & $0(0 \%)$ & $0(0 \%)$ & $47(62.7 \%)$ & $47(34.8 \%)$ \\
\hline high-grade & $0(0 \%)$ & $0(0 \%)$ & $31(41.3 \%)$ & $31(23 \%)$ \\
\hline \multicolumn{5}{|l|}{ Stage TNM } \\
\hline $\mathrm{Ta}$ & $0(0 \%)$ & $0(0 \%)$ & $33(44 \%)$ & $33(24.4 \%)$ \\
\hline $\mathrm{T} 1$ & $0(0 \%)$ & $0(0 \%)$ & $32(42.7 \%)$ & $32(23.7 \%)$ \\
\hline $\mathrm{T} 2$ & $0(0 \%)$ & $0(0 \%)$ & $12(16 \%)$ & $12(8.9 \%)$ \\
\hline T3 & $0(0 \%)$ & $0(0 \%)$ & $1(1.33 \%)$ & $1(0.73 \%)$ \\
\hline $\mathrm{T} 4$ & $0(0 \%)$ & $0(0 \%)$ & $0(0 \%)$ & $0(0 \%)$ \\
\hline CIS & $0(0 \%)$ & $0(0 \%)$ & $0(0 \%)$ & $0(0 \%)$ \\
\hline
\end{tabular}


are the first and third quartiles and the band inside the box is the second quartile (the median). The ends of the whiskers represent the lowest value within 1.5 interquartile range (IQR) of the lower quartile and the highest value within 1.5 IQR of the upper quartile. Any data not included between the whiskers represent outliers (small circles). Classification of the samples was made using a logistic regression model. Stepwise selection was used to determine which parameters are the most relevant for achieving best classification. Training of the model was carried out using the R software, which is a collection of machine learning and statistical tools. The performance of the model was tested by the leave-oneout cross-validation technique that uses the spectral data of various samples and also the known diagnostic group.

\section{Results}

Concentration matrices of synchronous spectra. For the analysis we chose the shift $\Delta \lambda=70 \mathrm{~nm}$ in synchronous fluorescence spectra which provides all excitation-emission bands of human urine. From these spectra CMSS were created. These matrices give us complete information about the concentration and fluorescence intensity of each sample (Figure 1). We tested all the parameters to find any statisti-

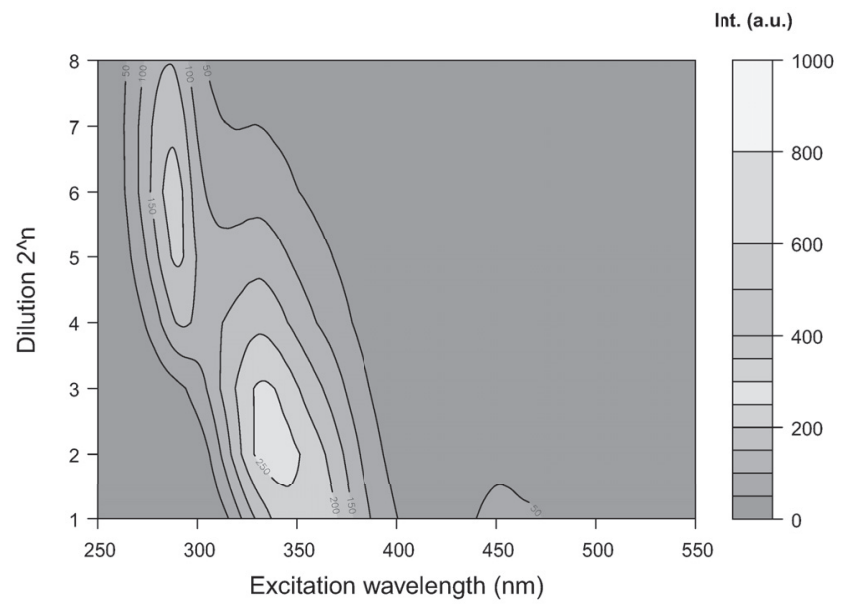

Figure 1. Concentration matrix of synchronous fluorescence spectra $(\Delta \lambda=70 \mathrm{~nm})$ of a representative urine sample. cally significant variables between the groups. According to the clinical practice we decided to compare the patients with hematuria, which are the patients that harbor the highest risk for bladder cancer.

We found several statistically significant differences in the fluorescence spectra of patients with hematuria and hematuria with tumor. The median, mean and interquartile range of these variables for both groups are given in Table 2 along with the $p$ values. The $p$-values of all variables are $<0.05$.

Parameters of CMSS analyzed. $I_{\max } 280$ - the maximal intensity of fluorescence at $280 \mathrm{~nm}$ wavelength. At this wavelength, we have observed a reduction of fluorescence intensity in the group of patients with tumor (Figure 2A). According to the published data [8], this fluorescence is attributed to tryphtophane metabolites, especially indoxylsulphate. Pix 250, a similar parameter to Imax 280 (sum of intensity from $250-300 \mathrm{~nm}$ wavelengths for all dilutions), gives us a broader picture in search for specific metabolites in this spectral area (Figure 2B). We know that the molar concentration of human urine has a huge variability. Ratio of intensities is a parameter that should mathematically minimize the differences in molar concentrations. Therefore, a statistically significant change in this parameter minimizes the risk attributable to differences only due to molar concentrations.

Parameters ratios. $\mathrm{I}_{\max } 280 / \mathrm{I}_{\max } 450$ parameter is a ratio of maximal intensities at $280 \mathrm{~nm}$ and $450 \mathrm{~nm}$ (Figure 2C). I280D128/I450D1 is a ratio of fluorescence intensity at $280 \mathrm{~nm}$ (dilution 1:128) and fluorescence intensity at $450 \mathrm{~nm}$ (dilution 1:1). I330D32/I450D1 is a ratio of fluorescence intensity at $330 \mathrm{~nm}$ (dilution 1:32) and fluorescence intensity at $450 \mathrm{~nm}$ (dilution 1:1) (Figure 2D).

Discrimination by receiver operator characteristic (ROC) curve. Figure 3 shows the ROC plot for the four significant fluorescent parameters. The cutoff values for each parameter and their respective sensitivity and specificity values are given in Table 3.

Histological grade. We have also tested spectroscopic changes related to the architecture of the tumor histological grade. We didn't find a statistically significant change that could predict a grade of the tumor (Figure 4). The highest differences between low and high group were observed at parameter $\mathrm{I}_{\max } 330$ where p-value reached 0.085 (Figure 4).

Table 2. Median, mean and interquartile range (IRQ) of the significant parameters used for statistical analysis along with their p-values (MannWhitney test).

\begin{tabular}{|c|c|c|c|c|c|c|c|}
\hline \multirow[b]{2}{*}{ Parameter } & \multicolumn{3}{|c|}{ Patients with HEMATURIA } & \multicolumn{3}{|c|}{ Patients with TUMOR } & \multirow{2}{*}{ p-value } \\
\hline & Median & Mean & IQR & Median & Mean & IQR & \\
\hline$I_{\max } 280$ & 246.9 & 294 & $159.9-391.1$ & 191.3 & 213 & $122.6-247.4$ & 0.0088 \\
\hline pix250 & 52550 & 65640 & $38430-81030$ & 44300 & 48490 & $31060-53490$ & 0.0155 \\
\hline $\mathrm{I}_{\max } 280 / \mathrm{I}_{\max } 450$ & 7 & 9.368 & $4.339-11.46$ & 4.248 & 5.654 & $2.833-6.537$ & 0.0010 \\
\hline I280D128/I450D1 & 3.329 & 5.803 & $2.361-5.546$ & 2.406 & 2.77 & $1.654-3.643$ & 0.0043 \\
\hline I330D32/I450D1 & 2.166 & 3.872 & $1.31-3.623$ & 1.518 & 2.103 & $1.113-2.318$ & 0.0155 \\
\hline
\end{tabular}

IQR - Interquartile range; p-value of Mann-Whitney test 
A

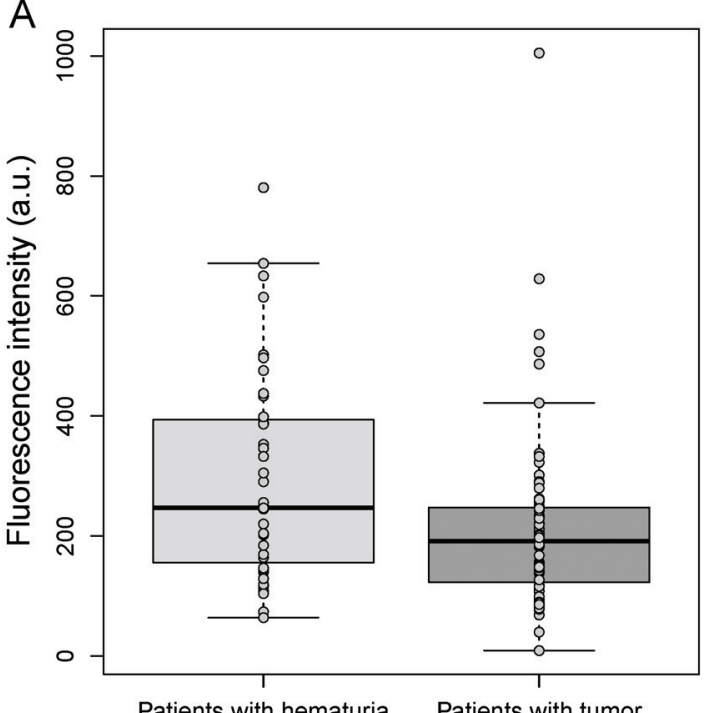

Patients with hematuria Patients with tumor
B

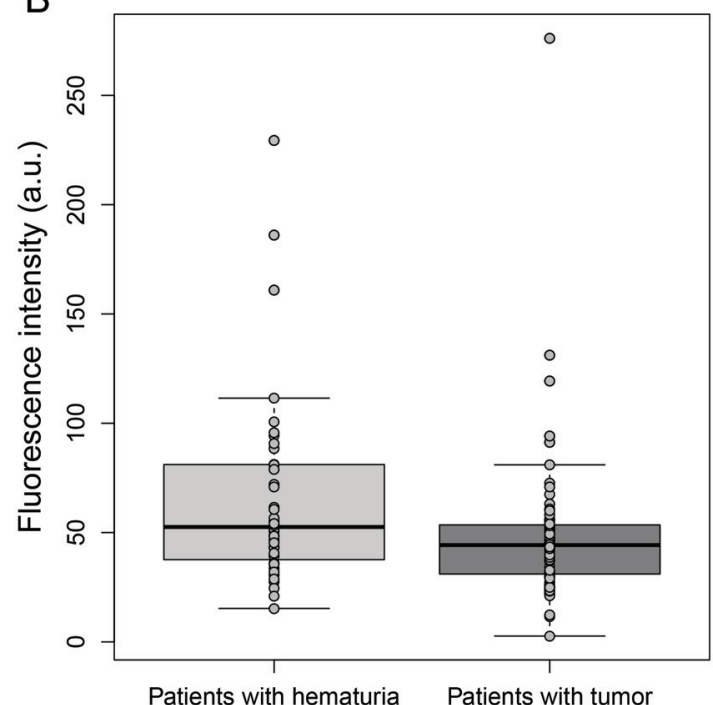

Patients with hematuria Patients with tumor

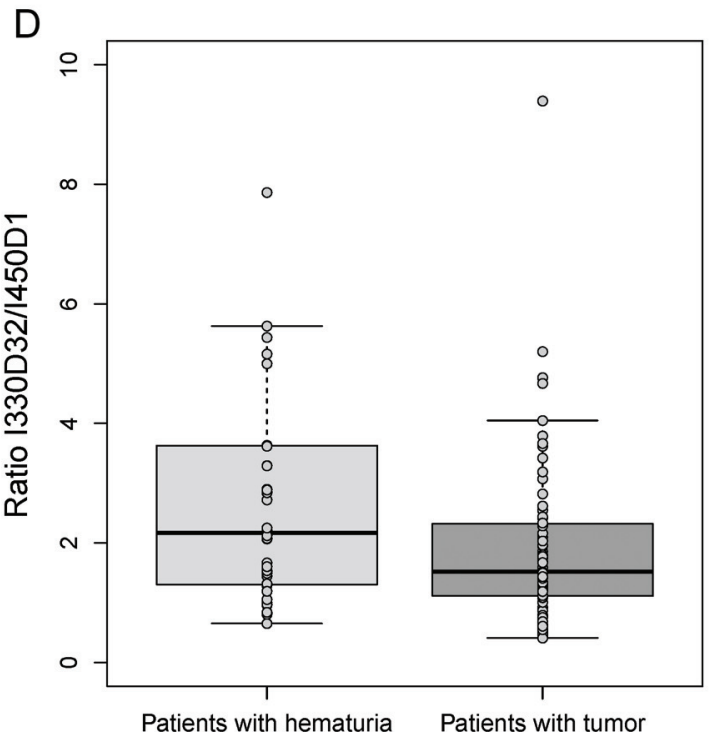

Figure 2. The box and whisker plot of A) maximum fluorescence intensity at $280 \mathrm{~nm}$ (Imax $280, \mathrm{p}=0.009$ ); B) fluorescence intensity sum from 250 to $300 \mathrm{~nm}$ for all dilutions (pix250, $\mathrm{p}=0.016$ ); C) maximum fluorescence intensity at $280 \mathrm{~nm}$ to $450 \mathrm{~nm}$ ratio (Imax280/ Imax450, $\mathrm{p}=0.001$ ); D) ratio of fluorescence intensity at $330 \mathrm{~nm}$ (dilution 1:32) and fluorescence intensity at $450 \mathrm{~nm}(\mathrm{I330D32} / \mathrm{I450D1}, \mathrm{p}=0.016)$ for patients with hematuria and patients with tumor.

Table 3. Cutoff value, area under curve (AUC) with $95 \%$ confidence interval (CI), sensitivity and specificity of the significant parameters.

\begin{tabular}{|c|c|c|c|c|}
\hline Parameter & CUTOFF VALUE & AUC (CI) & SPECIFICITY & SENSITIVITY \\
\hline$I_{\max } 280$ & 246.047 & $0.649(0.540-0.759)$ & $55.0 \%$ & $74.7 \%$ \\
\hline pix250 & 60324 & $0.638(0.527-0.748)$ & $40.0 \%$ & $85.3 \%$ \\
\hline $\mathrm{I}_{\max } 280 / \mathrm{I}_{\max } 450$ & 6.665 & $0.687(0.580-0.793)$ & $57.5 \%$ & $78.7 \%$ \\
\hline I280D128/I450D1 & 2.845 & $0.662(0.539-0.759)$ & $67.5 \%$ & $62.7 \%$ \\
\hline I330D32/I450D1 & 2.669 & $0.638(0.528-0.748)$ & $45.0 \%$ & $82.7 \%$ \\
\hline
\end{tabular}



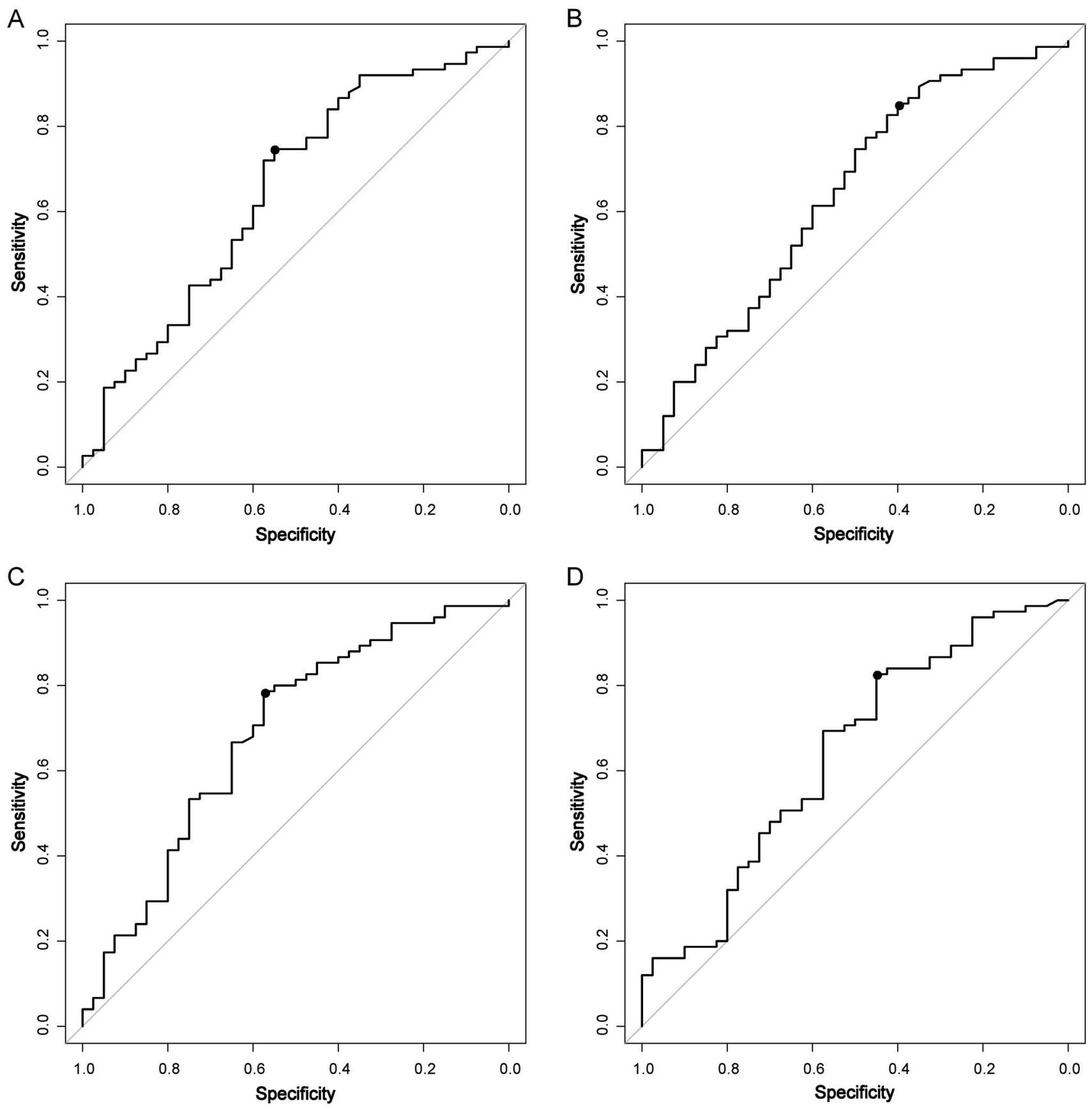

Figure 3. Receiver operating characteristics (ROC) curve analysis of A) maximum fluorescence intensity at $280 \mathrm{~nm}$ (Imax280); B) fluorescence intensity sum from 250 to $300 \mathrm{~nm}$ for all dilutions (pix 250); C) maximum fluorescence intensity at $280 \mathrm{~nm}$ to $450 \mathrm{~nm}$ ratio (Imax $280 /$ Imax450); D) ratio of fluorescence intensity at $330 \mathrm{~nm}$ (dilution 1:32) and fluorescence intensity at $450 \mathrm{~nm}$ (dilution 1:1). The black circle represents the optimal cutoff point.

Stage correlation. We found differences in maximal fluorescence intensity at $280 \mathrm{~nm}\left(\mathrm{I}_{\max } 280\right)$ in various stage groups (Kruskal-Wallis $\mathrm{p}=0.027$, Figure 5). In addition, we found a statistically significant correlation between the $\mathrm{I}_{\max } 280$ and stage of the disease (Spearman $\mathrm{rho}=-0.310$, $\mathrm{p}=0.007)$.
Cytological characteristics. All the patients involved in this study underwent cytological examination of urine. Cytology was performed according to recommended protocol and fixed with formaldehyde 1:1. Altogether 4 different pathologists reviewed the samples. From the entire cohort of patients (135 samples) we excluded 6 inconclusive 


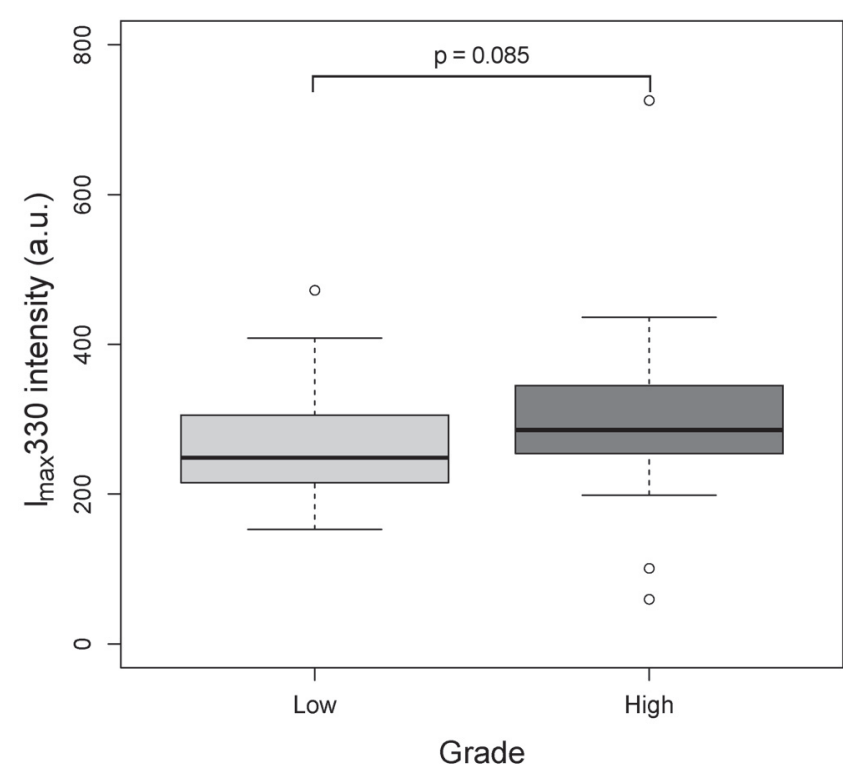

Figure 4. The box and whisker plot of maximum fluorescence intensity at $330 \mathrm{~nm}(\operatorname{Imax} 330)$ for different grades $(\mathrm{p}=\mathbf{0 . 0 8 5})$.

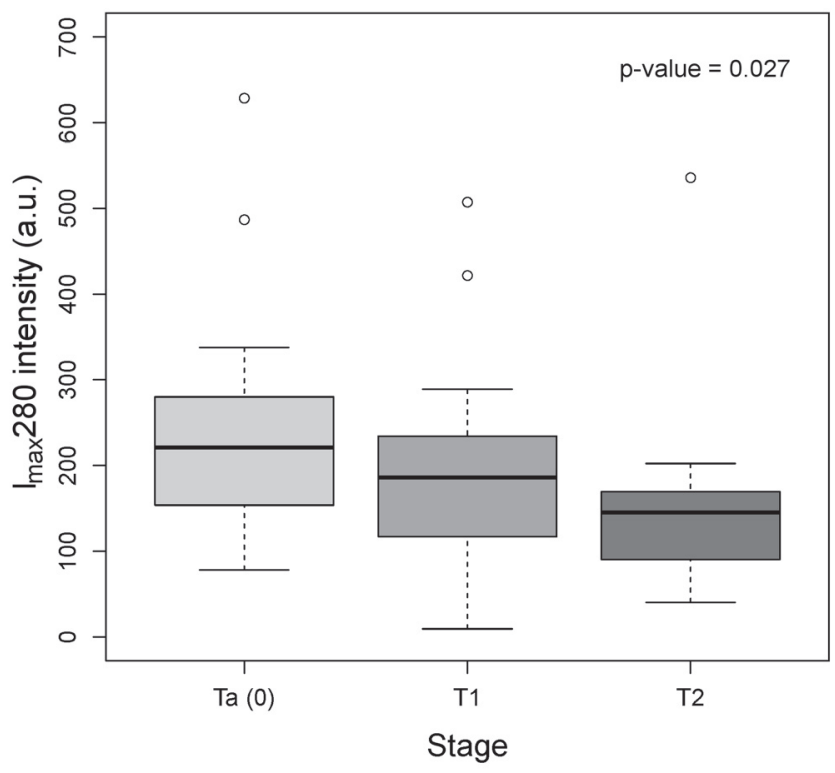

Figure 5. The box and whisker plot of maximum fluorescence intensity at $280 \mathrm{~nm}(\operatorname{Imax} 280)$ for different stages (Kruskal-Wallis $\mathrm{p}=\mathbf{0 . 0 2 7}$, Spearman rho $=-0.31, p=0.007)$.

cytologies which account for $4.44 \%$ of all tested samples. Sensitivity in the 2 groups of patients with hematuria was $57.97 \%$ and specificity $77.50 \%$ (Table 4 ). In the healthy cohort there were no positive cytologies. Grade correlation of cytologies was performed for the tumor group, which gave us similar results as we know from other studies [9]:

- low grade sensitivity $52.4 \%$ CI $95 \%(36.42-68 \%)$

- high grade sensitivity $66.7 \%$ CI 95\% (47.2-82.7\%)
Table 4. Classification of patients with hematuria and patients with bladder tumor by cytology.

\begin{tabular}{lcc}
\hline STATISTICS & Value & $\begin{array}{c}\text { 95\%confidence } \\
\text { interval (CI) }\end{array}$ \\
\hline Number of patients with hematuria & 40 & - \\
Number of patients with bladder tumor & 69 & - \\
Correctly classified & 71 & - \\
Incorrectly classified & 38 & - \\
Sensitivity & $57.97 \%$ & $45.48-69.76 \%$ \\
Specificity & $77.50 \%$ & $61.55-89.16 \%$ \\
Positive Predictive Value (PPV) & $81.63 \%$ & $70.73-89.10 \%$ \\
Negative Positive Predictive Value (NPV) & $51.67 \%$ & $43.61-59.63 \%$ \\
\hline
\end{tabular}

Table 5. Coefficient estimates for logistic regression model (AIC: 121.29).

\begin{tabular}{lcccc}
\hline Coefficients & Estimate & Std. Error & z value & $\operatorname{Pr}(>|\mathbf{z}|)$ \\
\hline Intercept & 1.4186 & 0.6865 & 2.066 & 0.038794 \\
Imax280 & -21.4056 & 6.6861 & -3.202 & 0.001367 \\
I280R8 & 18.0446 & 5.6051 & 3.219 & 0.001285 \\
I280R8/I450R1 & -102.8778 & 32.8192 & -3.135 & 0.00172 \\
Imax280/Imax450 & 22.6254 & 603960 & 2.708 & 0.006771 \\
Imax450 & -6.9172 & 3.8233 & -1.809 & 0.07042 \\
Cytology & 1.7281 & 0.4917 & 3.514 & 0.000441 \\
\hline
\end{tabular}

Table 6. Classification of patients with hematuria and patients with bladder tumor by logistic regression model with cytology.

\begin{tabular}{lcc}
\hline STATISTICS & Value & $\begin{array}{c}\text { 95\%confidence } \\
\text { interval (CI) }\end{array}$ \\
\hline Number of patients with hematuria & 40 & - \\
Number of patients with bladder tumor & 75 & - \\
Correctly classified & 89 & - \\
Incorrectly classified & 26 & - \\
Sensitivity & $77.33 \%$ & $66.21-86.21 \%$ \\
Specificity & $77.50 \%$ & $61.55-89.16 \%$ \\
Positive Predictive Value (PPV) & $86.57 \%$ & $78.16-92.07 \%$ \\
Negative Positive Predictive Value (NPV) & $64.58 \%$ & $53.76-74.10 \%$ \\
\hline
\end{tabular}

For the possible combination of spectroscopic analysis with cytology we performed a multivariable logistic regression model. Final regression model contains six parameters: $I_{\max } 280, I_{\max } 450$, I280D128 (intensity of fluorescence at $280 \mathrm{~nm}$, dilution 1:128), ratio I280D128/I450D1, ratio $I_{\max } 280 / I_{\max } 450$, and cytology. Estimated coefficients of model are shown in Table 5. This model involving cytology improved the sensitivity of either of the tests alone (Table 6).

\section{Discussion}

In our study we analyzed the possible role of spectroscopic examination of human urine in the diagnosis of bladder cancer, as $85 \%$ of the bladder tumor patients present with hematuria [10]. It is extremely important to 
have a non-invasive test that could distinguish between the hematuria patients.

At the spectroscopic analysis of urine samples, we have found statistically significant decrease of intensity peak at $280 \mathrm{~nm}$ and $330 \mathrm{~nm}$ in tumor patients. These are areas of fluorescence, which should refer to metabolites of tryptophan like indoxylsulphate for the $280 \mathrm{~nm}$ area which is part of the so called indol pathway of tryptophan metabolism and 3 hydroxyanthranillic acid for the $330 \mathrm{~nm}$ area, part of the kynurenin pathway $[8,10,11]$. Uptake and increased concentration of tryptophan metabolites in bladder cancer tissue could induce carcinogenesis [12]. The decreased intensity of these fluorescence areas in urine could be attributed to the accumulation of these substances in cancerous tissue. The precise identification of carcinogenesis is crucial for this understanding. The ratio testing of fluorescence areas also proved a significant increase in the $450 \mathrm{~nm}$ area. This represents the excitation area of flavonoids, which are known anticancer compounds and their increased excretion may indicate the lack of their availability in the body. Increase of fluorescence in this area was also observed by Masilamani et al. in patients with cervical cancer [13]. Spectroscopic urine analysis is a non-invasive test, that is cheap, easy and reproducible, therefore we think it has a potential role in diagnosis of bladder cancer. Cytology is the gold standard of non-invasive testing for bladder cancer. Our data show that it has a low sensitivity for low-grade tumors (52.4\%) and higher sensitivity for high-grade tumors $(66.7 \%)$. Our data are in concordance with other data from literature. Data from a meta-analysis of 1255 patients show a low sensitivity of cytology (34\%) with specificity of nearly $99 \%$ [14]. The data from our spectroscopic analysis show a higher sensitivity and lower specificity than cytology. Our spectroscopic urine analysis also found a significant decrease of maximal fluorescence intensity at $280 \mathrm{~nm}$ in correlation with the stage of the disease. This possible link needs to be validated in other studies. Similar results were published by other authors [7]. The results of the spectroscopic analysis could be compared with some other non-invasive urine tests like BTA STAT, nmP 22, Urovysion ${ }^{\circledR}$ or Immunocyt. These commercially available tests have comparable sensitivity and specificity to spectroscopic analysis. The fluorescence monitoring of human urine in hematuria patients could be an alternative in non-invasive diagnostics of bladder tumors. We have also performed this analysis in healthy controls without hematuria or tumor for control purposes, which has discovered multiple significant differences between the hematuria and healthy groups in fluorescence analysis. We did not include these results into our study, as we think that the risk of tumor is extremely low in patients without hematuria. It could be very interesting to see the analysis of fluorescence in patients with tumor and no hematuria, unfortunately the incidence of such tumors is low and we would not be able to include enough patients for statistical analysis. Therefore, a multi- center study of fluorescence analysis could give us more information. The combinations of the various non-invasive tests with spectroscopic analysis and cytology could be a promising perspective in non-invasive testing. The linear regression analysis of the combination of spectroscopy and cytology improved sensitivity of each of the tests alone. However, our results prove the fact that currently we don't have a non-invasive test that could alone or in combination reliably replace cystoscopy.

Acknowledgements: This work was financially supported by the Scientific Grant Agency of the Ministry of Education, Science, Research and Sport of the Slovak Republic and the Slovak Academy of Sciences VEGA 2/0092/16 and it is the result of the project implementation supported by the OPRaD funded by the ERDF (ITMS 26240120027).

\section{References}

[1] FERLAY J, SOERJOMATARAM I, DIKSHIT R, ESER S, MATHERS $C$ et al. Cancer incidence and mortality worldwide: Sources. methods and major patterns in GLOBOCAN 2012. Int J Cancer 2015; 136: E359-E386. doi: 10.1002/ ijc. 29210

[2] TRACZYK-BORSZYNSKA M, BORKOWSKA E, JABLONOWSKI Z, JEDRZEJCZYK A, PIETRUSINSKI $M$ et al. Genetic diversity of urinary bladder cancer and the risk of recurrence based on mutation analysis. Neoplasma 2016; 63: 952-960. doi: 10.4149/neo_2016_614

[3] SHARIAT SF, KARAM JA, LERNER SP. Molecular markers in bladder cancer. Curr Opin Urol 2008; 18: 1-8. doi: 10.1097/MOU.0b013e3282f1c5c1

[4] OHKAWA H, OHISHI N, YAGI K. New metabolites of riboflavin appear in human urine. J Biol Chem 1983; 258: 56235628.

[5] LYCETTE RM, LESLIE RB. Fluorescence of malignant tissue. Lancet 1965; 286: 436.

[6] SHAPIRO A, GOFRIT ON, PIZOV G, COHEN JK, MAIER J. Raman Molecular Imaging: A Novel Spectroscopic Technique for Diagnosis of Bladder Cancer in Urine Specimens. Eur Urol 2011; 59: 106-112. doi: 10.1016/j.eururo.2010.10.027

[7] MARTINICKY D, ZVARIK M, SIKUROVA L, LAJDOVA I, HUNAKOVA L. Fluorescence analysis of urine and its potential for ovarian cancer screening. Neoplasma 2015; 62: 500-506. doi: 10.4149/neo_2015_060

[8] LEINER MJP, HUBMANN MR, WOLFBEIS OS. The total fluorescence of human urine. Anal Chim Acta 1987; 198: 13-23. doi: 10.1016/S0003-2670(00)85002-3

[9] REID MD, OSUNKOYA AO, SIDDIQUI MT, LOONEY SW. Accuracy of grading of urothelial carcinoma on urine cytology: an analysis of interobserver and intraobserver agreement. Int J Clin Exp Pathol 2012; 5: 882-91.

[10] VARKARAKIS MJ, GAETA J, MOORE RH, MURPHY GP. Superficial bladder tumor. Aspects of clinical progression. Urology 1974; 4: 414-20. 
[11] MASILAMANI V, VIJMASI T, AL SALHI M, GOVINDARAJ K, VIJAYA-RAGHAVAN AP et al. Cancer detection by native fluorescence of urine. J Biomed Opt 2010; 15: 057003. doi: $10.1117 / 1.3486553$

[12] CHUNG KT, GADUPUDI GS. Possible roles of excess tryptophan metabolites in cancer. Environ Mol Mutagen 2011; 52: 81-104. doi: 10.1002/em.20588
[13] MASILAMANI V, ALSALHI MS, VIJMASI T, GOVINDARAJAN K, RATHAN RAI R et al. Fluorescence spectra of blood and urine for cervical cancer detection. J Biomed Opt 2012; 17: 98001. doi: 10.1117/1.JBO.17.9.098001

[14] LOTAN Y, ROEHRBORN CG. Sensitivity and specificity of commonly available bladder tumor markers versus cytology: results of a comprehensive literature review and meta-analyses. Urology 2003; 61: 109-118. 